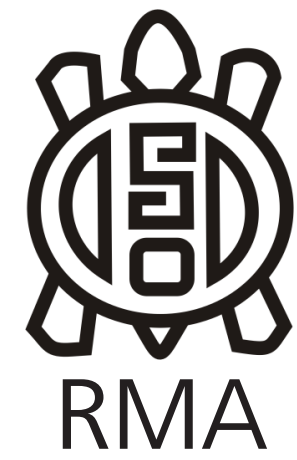

Museología

\title{
Consolidación del Estado Nacional Argentino a punta de lanza, sable y facón. Enfoque osteobiográfico e histórico de los restos del Cnel. Ambrosio Sandes (1815-1863)
}

\author{
Consolidation of the argentine national state at the tip of the spear, \\ saber and facón. Osteobiographical and historical approach to the \\ remains of the Col. Ambrosio Sandes (1815-1863) \\ P. Sebastián Giannotti*, Daniela A. Mansegosa*, \\ Horacio D. Chiavazza** y Emiliano J. Araujo ${ }^{* * *}$
}

\begin{abstract}
"CONICET; UNCuyo, Facultad de Filosofía y Letras, Instituto de Arqueología y Etnología, Laboratorio de Arqueología Histórica y Etnohistoria; Centro de Investigaciones Ruinas de San Francisco (Municipalidad de Mendoza). E-mail: pablosebastiangiannotti@gmail.com; alitmansegosa@gmail.com

* UNCuyo, Facultad de Filosofía y Letras, Instituto de Arqueología y Etnología, Laboratorio de Arqueología Histórica y Etnohistoria; Museo del Área Fundacional y Centro de Investigaciones Ruinas de San Francisco (Municipalidad de Mendoza).E-mail: hchiavazza@gmail.com

${ }^{* * *}$ UNCuyo, Facultad de Filosofía y Letras. E-mail: emilianojavara@gmail.com
\end{abstract}

\begin{abstract}
Resumen
La segunda mitad del siglo XIX estuvo caracterizado por la consolidación del Estado nacional argentino en el marco de dos cosmovisiones políticas-ideológicas contrapuestas: Liberalismo versus Federalismo. Las fuerzas militares $y$, especificamente, sus militares de alto rango cumplieron un rol trascendental al ejecutar el monopolio de la violencia desde los Estados Provinciales en su proyección nacional. El objetivo de este trabajo es estudiar, desde un enfoque osteobiográfico-histórico, un entierro secundario exhumado de la sepultura de Ambrosio Sandes (18151863) (Cementerio Municipal de Mendoza, Argentina) coronel unitario del Ejército Argentino cuya participación fue ampliamente documentada en los campos de batalla y en el escenario político nacional. A nivel esqueletal, se analizaron indicadores de estrés metabólico-nutricional, estrés funcional, patologías, traumas y salud oral. Los resultados permitieron elaborar un perfil osteobiográfico general y otro individualizante, brindando fundamentos que permitieron aproximarse a una posible identificación. Así mismo, se discuten los bioindicadores de acuerdo a las distintas etapas de su vida (infancia, carrera militar y los eventos próximos a su fallecimiento). De esta forma, se aporta no solo información osteobiográfica de uno de los personajes más relevantes del periodo, sino también permite vivenciar las lógicas del Estado en formación plasmada en el cuerpo de sus protagonistas.
\end{abstract}

Palabras clave: Ambrosio Sandes; Ejército Argentino; Guerra; Bioarqueología; Mendoza.

\begin{abstract}
The second half of the $19^{\text {th }}$ century was determined by the consolidation of the Argentine national state within the framework of two opposing political-ideological worldviews: Liberalism versus. Federalism. The military forces and, specifically, its high-ranking military played a transcendental role by executing the monopoly on violence from the Provincial States in their national projection. The aim of this work is to study, from an osteobiographical-historical approach, a secondary burial exhumed from the grave of Ambrosio Sandes (1815-1863) (Municipal Cemetery of Mendoza, Argentina) who was the unitary colonel of the Argentine Army with a documented participation in the battlefields and on the national political stage. At the skeletal level, analyze indicators of metabolic-nutritional stress, functional stress, pathologies, traumas and oral health. The results allowed to elaborate a general osteobiographical profile and another individualizing one, providing foundations that allowed to approximate a possible identification. Likewise, they discuss the bioindicators according to the different stages of their life (childhood, military career and the events near their death). In this way, not only osteobiographical information of one of the most relevant characters of the period is provided, but also allows to live the logic of the State in formation embodied in the body of its protagonists.
\end{abstract}

Keywords: Ambrosio Sandes; Argentine Army; War; Bioarchaeology; Mendoza. 


\section{Introducción}

La segunda mitad del siglo XIX estuvo caracterizado por la consolidación del Estado nacional argentino en el marco de dos cosmovisiones políticas-ideológicas contrapuestas: Liberalismo versus Federalismo. Las fuerzas militares, de distinta naturaleza (Ejército de Línea, milicias provinciales, fuerzas regulares e irregulares), cumplieron un rol trascendental al ejecutar el monopolio de la violencia desde los Estados Provinciales en su disciplinamiento social interno como así también en la proyección hacia la consolidación de un Estado Nacional (Garavaglia, Ruiz y Zimmermann, 2012). En este contexto se destaca la participación del Cnel. Ambrosio Sandes, uno de los militares que mayor transcendencia tuvo por su historial de victorias en los campos de batallas y la crueldad con que trataba a los prisioneros federales (Mercado Luna, 1974; Sarmiento, 2014 [1865]).

En el año 2017, en el marco de estudios de impacto arqueológico y diagnóstico del estado de conservación de algunas estructuras del Cementerio Municipal de la Capital de Mendoza (Argentina), realizados por el equipo del Centro de Investigaciones Ruinas de San Francisco (CIRSF), se tuvo la oportunidad de exhumar los restos de Ambrosio Sandes. La intervención, realizada bajo aprobación del municipio, tenía dos finalidades. En primer lugar, la recuperación y conservación de los restos, ya que la estructura presentaba altos niveles de humedad y estaba deteriorando los huesos. En segundo lugar, realizar el análisis bioarqueológico de los mismos para determinar si los restos corresponden con el individuo referenciado en la sepultura, ya que se han registrado para el mismo periodo discordancia entre los registros de individuos inhumados en el cementerio con los efectivamente hallados (Mansegosa, Zorrilla, Prieto Olavarría y Giannotti, 2012).

En este sentido, se valió del estudio de los restos óseos y dentales humanos para la reconstrucción del perfil osteobiográfico lo que supone la determinación del sexo, la estimación de la edad, de la estatura, de la ascendencia biológica, así como también la identificación de patologías, traumas, uso del cuerpo, salud oral, entre otros. El conjunto de estos datos ofrece información sobre la interacción entre el individuo y el ambiente (Neves, 1984). Dicha información biológica puede ser combinada con la evidencia documental, histórica y etnográfica para poder otorgarles significado social a aquellos indicadores óseos que dan cuenta de la historia de vida (Glencross, 2011). En esta línea, el individuo es considerado un agente activo, que está inmerso y es parte de un contexto socio-histórico, donde los cambios óseos experimentados son resultado de múltiples factores biológicos, sociales y psicosociales (Elder, Johnson y Crosnoe, 2003).

El objetivo de este trabajo es presentar los resultados del análisis osteobiográfico de los restos óseos correspondientes a un entierro secundario recuperado de la sepultura del Cnel. Ambrosio Sandes (1863), ubicada en el sector histórico del Cementerio de la Capital de Mendoza. Los indicadores óseos y dentales se interpretan en relación con la evidencia documental e histórica desde un enfoque multidisciplinario.

\section{Biografía de Ambrosio Sandes}

Sandes nació en el año 1815 en el seno de una familia campesina regularmente acomodada del Departamento de Paysandú, Banda Oriental (actual Uruguay). Sus padres fueron don Pablo Sandes, de ascendencia europea, y doña Sabina Ruiz Díaz, de origen nativo o mestizo. Su infancia se desarrolló en la campaña, donde su familia se dedicaba al pastoreo y comercialización de ganado vacuno. De niño montaba a caballo y colaboraba en las tareas agrícolas y de pastoreo arreando ganado de la estancia a la ciudad. Llegó a destacarse entre sus vecinos por la fuerza y valor con tan solo 14 años. A los 19 años, cuando tuvo lugar la invasión de los ejércitos rosistas al mando de Manuel Oribe, huyó al monte junto a otros orientales para evitar servir al ejército. Sin embargo, su padre lo obligó a enlistarse iniciando así su carrera militar en la década de 1830 (Cuestas, 1897).

En poco tiempo ascendió de soldado a comandante, participó de decenas de batallas bajo las órdenes de los colorados durante la Guerra Grande de la Banda Oriental (1839-1851) y protagonizó numerosas escaramuzas con milicianos, soldados y bandidos rurales, resultando gravemente herido en muchas de ellas. En 1852 Sandes combatió en la batalla de Caseros como integrante del Ejército Grande, al mando del general Justo José de Urquiza, y participó en el sitio a Buenos Aires a fines del mismo año (donde se registraron enfrentamientos diarios). Tras volver a Uruguay, donde recibió el rango de Teniente Coronel, es destituido del cargo y estuvo dos años (1856-1858) al margen de la actividad bélica. Durante este tiempo, contrajo matrimonio, tuvo hijos legítimos y se dedicó a actividades ganaderas (arreo de ganado) y agrícolas (siembra de trigo) (Cuestas, 1897). En 1858 debe exiliarse y lo recibe Urquiza como asilado político en Entre Ríos (Saldaña, 1939). Para este entonces, es descripto como un hombre donde:

"Predominaba en él la raza blanca, á que pertenecía por su padre (que era hijo de españoles), de elevada estatura, de pelo y barba castaños (usaba la barba entera), con largos bigotes, frente despejada, cara angulosa, nariz aguileña, cabeza desarrollada, y ojos negros, vivos y fuertes" (Cuestas, 1897:400).

A partir de este momento, su vida se desarrolló por completo en suelo argentino, convirtiéndose en uno de los militares más eficaces, útiles y crueles del Gral. Bartolomé Mitre, quien fuera primero Gobernador de la Provincia de Buenos Aires y luego presidente de la República Argentina (1862-1868) (Mercado Luna, 1974). La primera orden que 
recibe Sandes de Mitre fue la creación de una caballería organizada y regular (Guías de Buenos Aires) (Cuestas, 1897), la cual participó en el enfrentamiento entre el Estado de Buenos Aires y la Confederación Argentina en la batalla de Cepeda (1859) y Pavón (1961). Mientras que Sandes sale herido gravemente de la primera (al punto de creer que estaba muerto), participaría activamente de la segunda (Mercado Luna, 1974). Con el triunfo de Pavón, Mitre asume el título de Encargado del Poder Ejecutivo Nacional e implementa, mediante las fuerzas porteñas nacionalizadas, una política de pacificación hacia el interior del país. De esta forma, hace uso del monopolio de la violencia legítima como condición necesaria para la consolidación del Estado nacional y del liberalismo como eje ideológico rector (Codesido, 2016).

En este contexto, Sandes y su $1^{\circ}$ Regimiento de Caballería de Línea protagonizó múltiples escaramuzas de menor escala y algunas batallas trascendentales en menos de dos años, llegando a recorrer en caballo 200 leguas en 10 días (Cuestas, 1897:415), lo que equivale a un promedio de 100 kilómetros por día. Entre 1861 y 1863 , se destaca su participación en las batallas en Santa Fe (Cañada de Gómez), La Rioja (Las Aguaditas de los Valdeses y Lomas Blancas) y Córdoba (Las Playas), donde torturó y mató (a lanzazos, fusilamientos y degüellos) a más de 1000 prisioneros federales; en muchos casos, cremó los cuerpos para que no puedan ser enterrados bajo cristiana sepultura, dando origen a la "carbonera de Sandes" (Chávez, 1975; Mercado Luna, 1974; Sarmiento, 2014 [1865]). Sandes alcanzó renombre no solo por la violencia con que trataba a enemigos y subalternos, sino también por la intensidad de sus enfrentamientos durante el combate. La violencia impresa en su cuerpo se plasmó en las 53 heridas contabilizadas por Sarmiento, quien afirmaba: "... el cuerpo de Sandes atraía los misiles; su alta figura las venganzas, como las agujas de los templos atraen los rayos. En tiroteos parciales de avanzadas, Sandes salía herido siempre" (Sarmiento, 2014 [1865]:139). Sus cicatrices respondían a facón, puñal, lanza, estoque y sable (Cuestas, 1897), a lo que Sarmiento (2014 [1865]) agrega los impactos de proyectil. Este último, detallaba la cuantificación de las heridas del "Cid campeador de las pampas" (Sarmiento, 2014 [1865]):

"El boletín del ejército llevaba cuenta de sus heridas. En un tiroteo en la campaña de Buenos Aires, una bala en el estómago, cuarenta y nueve heridas hasta entonces. En el Carcarañá la quincuagésima, de bala, en la caja del cuerpo quince días después. La quincuagésima prima, puñalada de un asesino en el pecho en San Luis; la quincuagésima segunda un balazo después de la paz, paseándose a los alrededores de su campamento en los Llanos. La quincuagésima tercera, una lanzada en una pierna en las Lomas Blancas" (Sarmiento, 2014 [1865]:140).

Las descripciones de los contemporáneos argentinos para estas fechas se refieren a él como un hombre:
"Corpulento, de piel blanca, de pelo y barba castaños; tiene un gesto huraño y bravío; es silencioso, casi taciturno, amigo de pocas palabras y enemigos de las bromas; a la menor contrariedad se encabrita. Son terribles sus arranques de furor. Su tropa le teme y él se complace en mantener ese temor" (Mercado Luna, 1974:99).

Si bien hay imprecisiones sobre el día de muerte, se sabe que fue en 1863 en Mendoza, entre el 5 de septiembre (según la placa de su sepultura) y el 15 de septiembre (Campano, 2017). Las circunstancias fueron extrañas, generando un debate historiográfico en torno a la causa de su muerte. Mientras algunos lo asocian a un envenenamiento por gente del Chacho Peñaloza en el marco de un banquete de gala con dirigentes políticos mendocinos (Campano, 2017), otros lo adjudican a lesiones generadas días antes en la provincia de San Luis por un enfrentamiento a la salida de una pulpería (Quiroga, 1975). Sus restos fueron inhumados dos días después de su muerte en el Cementerio Municipal de la Capital de Mendoza (Campano, 2017).

\section{Materiales y métodos}

La exhumación de los restos óseos se realizó priorizando conservar la integridad estructural de la sepultura. La misma es un cipo, definido como columna o pilastra sin base que contiene al difunto dentro de la estructura funeraria (Sosa, 2015). Presenta una forma prismática con base rectangular de $3 \mathrm{~m}$ de alto por 1,5 $\mathrm{m}$ de ancho. Hacia la pared oeste, donde se había desprendido la mayor cantidad de revoque y estaban expuestos los ladrillos, se observó un cambio en el patrón de la disposición de los mismos (de canto) en un área restringida de 40 $\mathrm{cm}$ de alto por $40 \mathrm{~cm}$ de ancho, lo que sugería una modificación de la pared original. Se procedió a retirar cuidadosamente la menor cantidad de ladrillos para observar hacia el interior sin afectar la integridad de la estructura. Se hallaron escombros de ladrillo, argamasa, fragmentos de molduras y una placa de mármol que no presentaba ningún tipo de inscripción. Tras retirar todos los materiales constructivos, se pudo acceder al cajón el cual se encontraba desarmado debido a que la madera estaba en proceso de desintegración, y en su interior contenía los restos humanos. Se retiró cuidadosamente cada elemento de la sepultura, separando el material bioantropológico del resto de materiales arqueológicos. Se tomaron muestras de tejido seco, cabello y depósito de cristales minerales para futuros análisis. Los huesos fueron dispersados sobre zarandas en un lugar ventilado con la finalidad de reducir los altos niveles de humedad que tenían y acondicionados in situ mediante limpieza con cepillado suave y levemente humedecido, embolsado y rotulado. Posteriormente, los restos fueron trasladados al laboratorio del Centro de Investigaciones Ruinas de San Francisco (Área Fundacional, Municipalidad de Mendoza) para su análisis. 
En el laboratorio, se realizó el análisis bioantropológico donde se determinó el tipo y lateralidad de cada elemento óseo y pieza dental. Luego se analizaron variables tafonómicas: estado general de conservación, grado de integridad por elemento, presencia de marcas de raíces, roedores, depósitos de carbonato, óxido de manganeso y fracturas postmortem (Fernández-Jalvo y Andrews, 2016).

Para la determinación del sexo, se relevaron rasgos morfológicos del cráneo (proyección de la cresta nucal, tamaño del proceso mastoides, robusticidad de la glabela, espesor del margen supra orbital y proyección de la eminencia mentoniana) y de la pelvis (arco ventral, concavidad subpúbica, la superficie de la rama isquiopúbica, la escotadura ciática y el surco preauricular) (Buikstra y Ubelaker, 1994; Krenzer, 2006). También se relevaron variables morfométricas del fémur y del húmero y se aplicaron las fórmulas discriminantes de Pearson (1919 en Bass, 1995) y de Stewart (1970 en Byers, 2002) respectivamente. Adicionalmente, se aplicaron funciones discriminantes desarrolladas para poblaciones locales del periodo colonial (siglos XVII-XIX) (Mansegosa, Giannotti, Chiavazza y Barrientos, 2018).

Para estimar la edad de muerte se analizaron cambios degenerativos de la superficie auricular del ilion (Meindl y Lovejoy, 1989 en Krenzer, 2006; Lovejoy et al., 1985 en Krenzer, 2006) y de la sínfisis púbica (Suchey y Brooks, 1990 en Krenzer, 2006), el grado de desarrollo y erupción dentaria (Buikstra y Ubelaker, 1994), el grado de fusión de las epífisis (Buikstra y Ubelaker, 1994; Krenzer, 2006), los cambios morfológicos del extremo esternal de la cuarta costilla (Byers, 2001 en Krenzer, 2006; Steele y Bramblett, 2000 en Krenzer, 2006), y la translucencia apical de la raíz de la dentición uniradicular (Lamendin et al., 1992).

La ascendencia biológica se estableció en base a las características morfológicas faciales del cráneo (Burns, 2012), odontológicas (Hillson, 2002) y al índice subtrocantérico del fémur (Gilbert y Gill, 1990). La estatura se calculó en base a la longitud máxima de húmero, radio, cúbito, fémur, peroné y tibia $(\mathrm{mm})$, que fueron medidos con una tabla osteométrica. Para esto se aplicaron las ecuaciones propuestas por Sjovold (1990). Se calculó la masa corporal promedio en vida del individuo en base a ecuaciones de regresión considerando el diámetro vertical de la cabeza femoral y el ancho bi-ilíaco (rearticulando los coxales y el sacro, y considerando la estatura calculada) (Auerbach y Ruff, 2004).

Se relevaron indicadores de dieta y salud oral: desgaste del esmalte dental, caries, cálculo dental, pérdida de piezas dentales antemortem, enfermedades periapicales y periodontitis (Buikstra y Ubelaber, 1994; Hillson 2002; Smith, 1984). Para los indicadores de estrés metabóliconutricional, se analizaron las hipoplasias del esmalte dental (Goodman y Rose, 1990) y la hiperostosis porótica y criba orbitalia del cráneo (Stuart-Macadam, 1985). Las patologías óseas fueron analizadas mediante observación escópica (Waldron, 2009; Weston, 2012) y empleo de radioscopia televisada (Dinar 500C-AF). Finalmente, se analizaron indicadores de estrés funcional a partir del relevamiento de la presencia e intensidad de cambios entésicos (CE) fibrosos (Hawkey y Merbs, 1995) y fibrocartilaginosos (Henderson, Mariotti, Pany-Kucera, Villotte y Wilczak, 2015); distribución e intensidad de enfermedad degenerativa articular (EDA) (Luna, Aranda y Amorim Alves, 2017), nódulos de Schmorl (NS) (Suby, 2014) y Osteocondritis Disecante (OD) (Zúñiga Thayer, Suby, Flensborg y Luna, 2018). Se evaluó la asimetría direccional de la porción apendicular a partir de la medición de proporciones métricas externas de diáfisis de huesos largos del húmero (índice diafisario del húmero), fémur (índice pilástrico y mérico) y tibia (índice cnémico) (Martin, 1957 en Del Rio Muñoz, 2000); presencia de fracturas premortem (Lovell, 2008; Waldron, 2009) y de marcadores mecánico posturales de facetas extras, impresiones y extensiones articulares (Estévez González, 2002), como así también el ángulo de torsión femoral (Wescott, Cunningham y Hunt, 2014).

\section{Resultados}

El análisis contextual del entierro confirma que se trata de una inhumación secundaria por la disposición desarticulada de los elementos óseos dentro del ataúd. Se recuperaron restos óseos y dentales del individuo, así como también algo de tejido blando seco adherido y pelo los cuales fueron separados para futuros análisis. Además, se hallaron fragmentos del textil (de vestimenta y de posible mortaja), un botón, restos de madera y herraje del ataúd, y un carozo de durazno.

El esqueleto estaba completo exceptuando la ausencia postmortem de huesos de la mano (6 falanges izquierdas y 7 derechas, un carpo derecho y uno izquierdo, y un metacarpo izquierdo) y del pie (7 falanges izquierdas y 8 derechas). Desde el punto de vista tafonómico, todos los elementos tenían un grado de completitud elevado (75-100\%), con fracturas postmortem en el 6,7\% $(n=10 / 150)$ de elementos presentes (cotillas, cráneo, escápulas y pérdida de tejido cortical en ilion izquierdo). En términos generales, la conservación es regular ya que presenta agrietamiento del tejido cortical con bordes curvados en huesos largos. El cráneo estaba más afectado debido a su estado frágil y con desprendimiento de tabla externa en forma de láminas. A su vez, tenía depósitos de cristales minerales con una distribución uniforme en toda la superficie. Los elementos no presentaban marcas de raíces, roedores, manchas de óxido de manganeso ni marcas de excavación. El 9,3\% ( $n=14 / 150)$ de elementos presentaban depósitos de carbonato siguiendo un patrón uniforme y leve.

El perfil osteobiográfico general indica que se trata de un individuo masculino con un rango de edad entre 41 
y 51 años. Tiene una estatura promedio de 177,4 cm y una masa corporal promedio en vida de $70 \mathrm{~kg}( \pm 4 \mathrm{~kg})$. Su ascendencia biológica no es concluyente ya que las características morfológicas faciales lo posicionan como europeo mientras que el índice subtrocantérico $(72,88)$ lo ubica como nativo americano.

El aparato oral indica, en apariencia macroscópica, ausencia de caries y de procesos periapicales. Pero presenta periodontitis en todas las piezas dentales de la mandíbula, cálculo dental en el maxilar (leve, en cuello y raíz de cara vestibular de todos los dientes) y mandíbula (leve, en corona y cuello de cara vestibular de dentición anterior de todos los dientes). El patrón de desgaste es plano, con un grado moderado (dentición anterior) y leve (posterior). Presenta pérdida de piezas dentales postmortem en el 31,3\% ( $n=10 / 32)$ de alveolos (seis del maxilar y cuatro de la mandíbula) y ninguna premortem.

Se detectaron cuatro líneas de hipoplasias del esmalte dental en la superficie bucal del 13,6\% (n=3/22) de las piezas dentales presentes (canino inferior izquierdo, el homólogo derecho y el canino superior derecho). De ellos, ambas líneas de los caninos inferiores son simétricas por lo que corresponde a un evento de tipo sistémico producido en torno a los cuatro años y medio, mientras que el canino superior derecho presenta dos líneas producidas a los tres y a los dos años y medio (Goodman y Rose, 1990). No presentaba lesiones de hiperostosis porótica ni criba orbitalia.

El resquebrajamiento del tejido cortical debido a procesos tafonómicos dificultó la observación de las lesiones. A pesar de ello, se registraron reacciones periósticas (Weston, 2012) en el 4\% $(n=6 / 150)$ de los elementos presentes (Fig. 1): costilla izquierda (Fig. 1.1), metatarso izquierdo (Fig. 1.2), cúbito izquierdo (Fig. 1.3), tibia izquierda (Fig. 1.4), peroné izquierdo (Fig. 1.5) y peroné derecho (Fig. 1.6). Todas las lesiones estaban reabsorbidas con excepción del metatarso de mano izquierda (en estado activo de acuerdo a la descripción de Weston, 2012:500).

En la región frontal del cráneo (por encima de la órbita izquierda), se detectó una lesión ósea proliferativa en forma de láminas superpuestas junto a erosión cortical lítica de los bordes. Dicha alteración presenta una forma ovalada con un diámetro de $40 \mathrm{~mm}$ y que se proyecta $5 \mathrm{~mm}$ desde la superficie cortical de la tabla externa. La descripción y ubicación de este tipo de lesión ósea es compatible con el diagnóstico de Hemangioma (intraóseo o intracortical) (Haeren, Dings, Hoeberigs, Riedl y Rijkers, 2012; Nasser et al., 2007; Vega et al., 2010) (Fig. 2). Es importante mencionar que toda la bóveda craneana e inclusive el sector donde se encuentra el tumor, está afectado por el agrietamiento postmortem del cortical.

Se registró la presencia de CE, EDA, NS y OD (Fig. 3). LoS
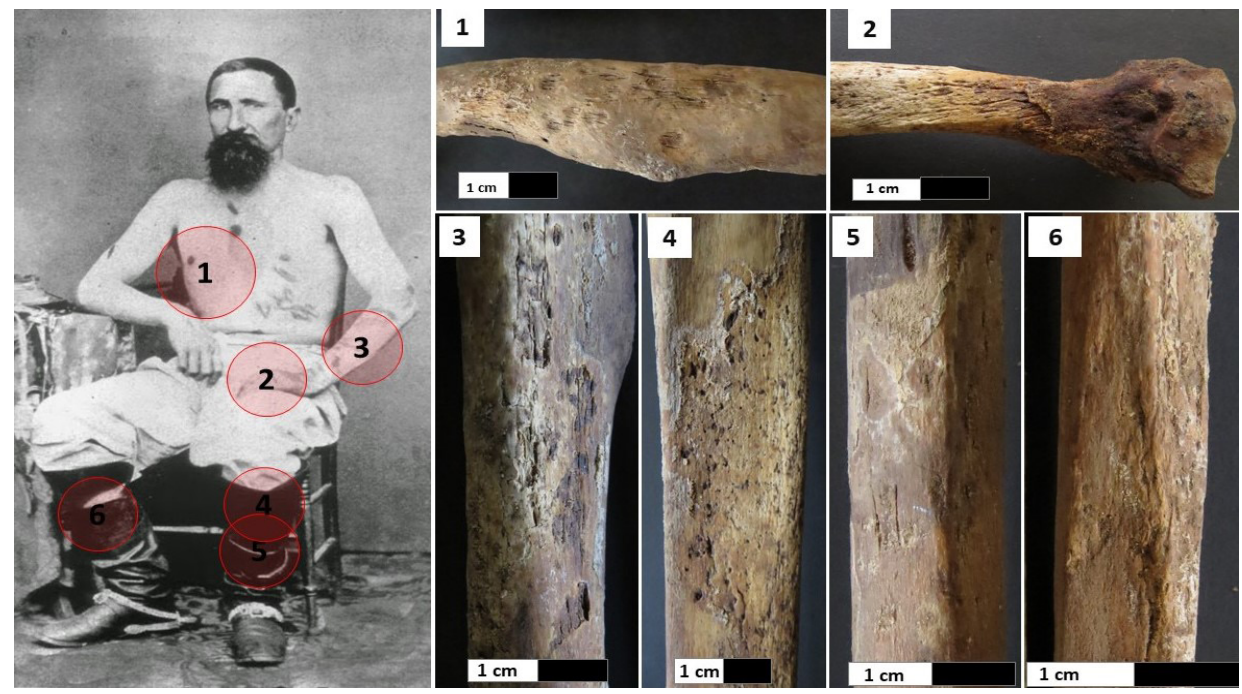

Figura 1. Ubicación de elementos con periostitis sobre daguerrotipo de Ambrosio Sandes, tomado por Emil Mangel Du Mesnil en 1861, donde se lo ve con el torso desnudo luciendo sus heridas (Cuarterolo y Alexander 2001). Referencia: 1, superficie dorsal de cuerpo de costilla derecha (entre la $4^{\circ}$ y $7^{\circ}$, identificación no precisada); 2 , metáfisis proximal del $2^{\circ}$ metacarpo izquierdo; 3 , tercio diafisiario proximal de cúbito izquierdo; 4, tercio diafisiario distal de tibia izquierda; 5 , tercio diafisiario proximal de peroné izquierdo; 6; tercio diafisiario proximal de peroné derecho.

Figure 1. Location of elements with periostitis on a daguerreotype of Ambrosio Sandes, taken by Emil Mangel Du Mesnil in 1861. He is seen with a upper body showing his wounds (Cuarterolo and Alexander 2001). Reference: 1, dorsal surface of the body of the right rib (4th and 7th, identification not specified); 2, proximal metaphysis of the 2nd left metacarpus; 3, proximal diaphyseal third of left ulna; 4, distal diaphyseal third of left tibia; 5, proximal diaphyseal third of left fibula; 6; proximal third diaphyseal of the right fibula. 

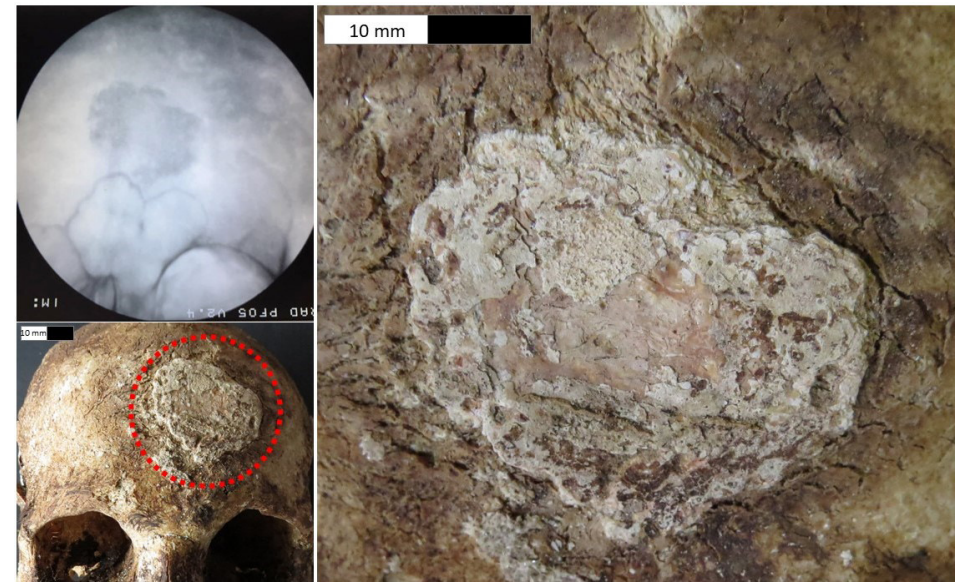

Figura 2. Detalle (macroscópico y radioscópico) de neoplasia en cráneo (hemangioma), caracterizada por una lesión ósea proliferativa con actividad osteolítica (erosión) en tejido cortical de la región frontal.

Figure 2. Detail (macroscopic and radioscopic) of a skull neoplasia (hemangioma), characterized by a proliferative bone lesion with osteolytic activity (erosion) in cortical tissue in the frontal region.
$C E$, registrados en el 31,1\% $(n=33 / 106)$ de las entesis relevadas, presentan mayor desarrollo asimétrico en la clavícula derecha (ligamento costoclavicular con lesión de estrés leve y $m$. deltoides -inserción- con osificación moderada) (Fig. 3.1 y 3.2), el húmero izquierdo (tendón común de los flexores y extensores en epicóndilo medial y lateral respectivamente con formación ósea severa en zona 1 y zona 2 de ambas entesis) (Fig. 3.3), la $8^{\circ}$ costilla derecha ( $m$. intercostales -origen- en margen inferior con osificación severa) (Fig. 3.5), el cúbito izquierdo (M. supinador con robusticidad grado leve) y el húmero derecho (M. deltoides -origen- con robusticidad grado leve). Además, presentaba osificación en apófisis espinosa del arco neural de la T6 y T7 $(m$. trapecio -origen- con osificación moderada) (Fig. 3.4), el isquion de ambas lateralidades ( $m$. semimembranoso -origen- en tuberosidad isquiática de con formación ósea severa en zona 1 y 2, y erosión en zona 2) (Fig. 3.6) y en la tuberosidad calcáneo lateral de ambas lateralidades (ligamento peroneocalcaneo con robusticidad grado moderado) (Fig. 3.7).

En cuanto a la EDA, el 6,1\% $(n=10 / 164)$ de las superficies analizadas presentaba proliferaciones periarticulares (osteofitos y labiado), lo que representa el 19,2\% $(\mathrm{n}=19 / 99)$ del total de elementos relevados. En la porción
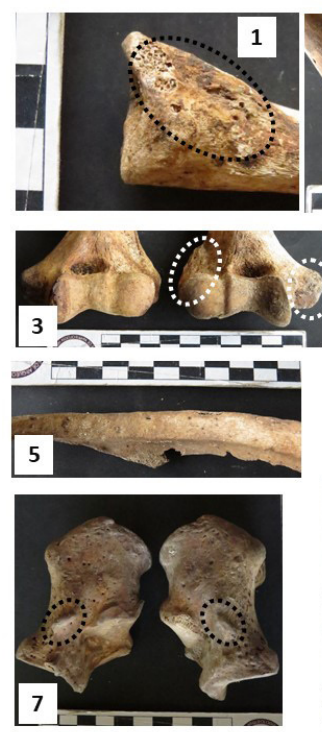
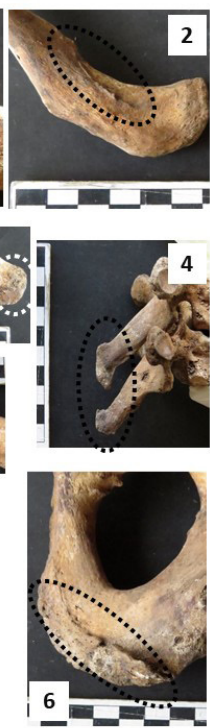
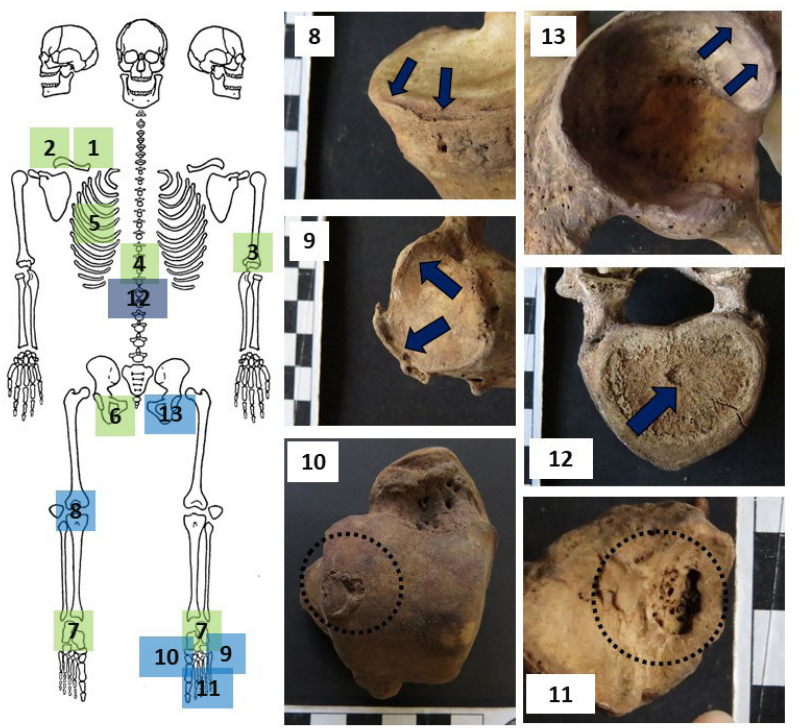

Figura 3. Indicadores de estrés funcional: CE, EDA, NS y OD. Referencias: 1, CE de ligamento costoclavicular en clavícula derecha; 2, CE de $m$. deltoides en clavícula derecha; 3, CE de epicóndilo medial (tendón común de los flexores) y lateral (tendón común de los extensores) en húmero izquierdo; 4, CE de $m$. trapecio en T6 y T7; 5, CE de $m$. intercostales en $8^{\circ}$ costilla derecha; 6 , CE de m. semimembranoso en hemipelvis derecha; 7, CE en ligamento peroneocalcaneo de calcáneos; 8, EDA leve en tibia izquierda; 9 , EDA moderada en calcáneo izquierdo; 10, OD en astrágalo izquierdo; 11, OD en $1^{\circ}$ metatarso izquierdo; 12, NS en T12; 13, EDA leve en acromion de hemipelvis izquierda.

Figure 3. Indicators of functional stress: CE, EDA, NS and OD. References: 1, CE of costoclavicular ligament in right clavicle; 2 , CE of $\mathrm{m}$. deltoid on right clavicle; 3, CE of medial epicondyle (common flexor tendon) and lateral (common extensor tendon) in left humerus; 4, CE of $m$. trapezius at T6 and T7; 5, CE of m. intercostals at 8th right rib; 6, CE of m. semimembranosus in right hemipelvis; 7, CE in calcaneal peroneocalcaneal ligament; 8, mild EDA in left tibia; 9, moderate EDA in the left calcaneus; 10, RE in left talus; 11, RE in left 1st metatarsal; 12, NS at T12; 13, mild EDA in left hemipelvis acromion 


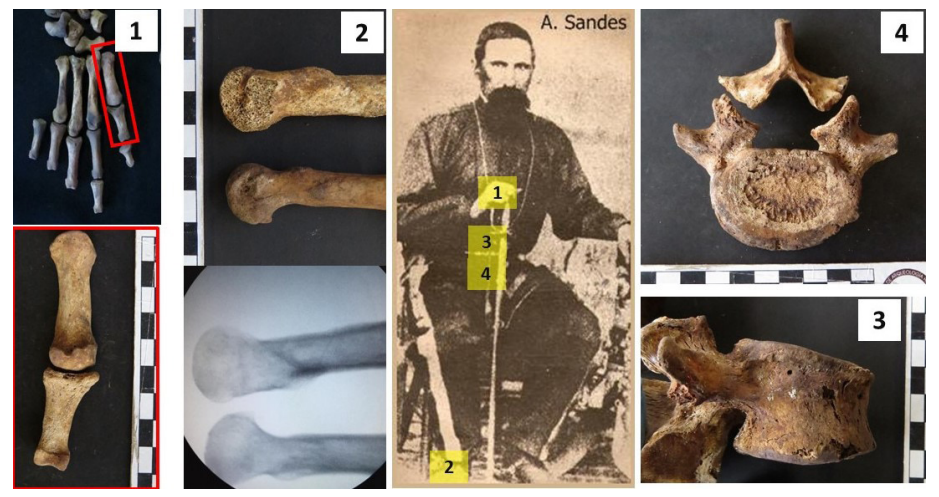

Figura 4. Ubicación de fracturas premortem, extensión de carilla articular y espondilólisis sobre daguerrotipo de Sandes (1863) posando con su espada (Chávez 1975:67). Referencias: 1, extensión de carilla articular $2^{\circ}$ falange derecha del pulgar; 2 , callos óseos en $2^{\circ}$ y $3^{\circ}$ metatarso derecho; 3, fractura por compresión en cuerpo vertebral de L2; 4, espondilólisis en L5.

Figure 4. Location of premortem fractures, joint facet extension and spondylolysis on a daguerreotype of Sandes (1863) posing with his sword (Chávez 1975: 67). References: 1, extension of the articular facet 2nd right phalanx of the thumb; 2, bony calluses in the 2nd and 3rd right metatarsals; 3, compression fracture in vertebral body of L2; 4, spondylolysis at L5.

Tabla 1. Índices diafisiarios externos, ángulo de torsión femoral y asimetría direccional. Referencia: $A D$, asimetría direccional (diferencia en el valor nominal - $n$ - y porcentual -\%-); IDH, índice diafisiario del húmero; IP, índice pilástrico; IM, índice mérico (o subtrocantérico); IC, índice cnémico; ATF, ángulo de torsión femoral.

Table 1. External shaft indices, femoral torsion angle, and directional asymmetry. Reference: $A D$, directional asymmetry (difference in nominal value - $n$ - and percentage - $\%$-); IDH, diaphyseal index of the humerus; PI, pylastric index; MI, meric (or subtrochanteric) index; $\mathrm{Cl}$, cnemic index; ATF, femoral torsion angle.

\begin{tabular}{|c|c|c|c|c|c|}
\hline \multirow{2}{*}{ Elemento } & \multirow{2}{*}{ Indices y ATF } & \multicolumn{2}{|c|}{ Lateralidad } & \multicolumn{2}{c|}{ AD } \\
\cline { 3 - 6 } & & $\mathrm{D}$ & $\mathrm{I}$ & $\mathrm{n}$ & $\%$ \\
\hline Húmero & $\mathrm{IDH}$ & 78,6 & 79,6 & 0,9 & $1,2 \%$ \\
\hline \multirow{2}{*}{ Fémur } & $\mathrm{IP}$ & 118,3 & 116,3 & 2,0 & $1,7 \%$ \\
\cline { 2 - 6 } & $\mathrm{IM}$ & 72,5 & 84,0 & 11,4 & $13,6 \%$ \\
\hline Tibia & $\mathrm{IC}$ & 83,3 & 80,0 & 3,3 & $3,9 \%$ \\
\hline Fémur & ATF & $16^{\circ}$ & $18^{\circ}$ & $2^{\circ}$ & $11,1 \%$ \\
\hline
\end{tabular}

axial, se registraron proliferaciones periarticulares en la columna vertebral, donde el 33,3\% $(n=8 / 24)$ de las vértebras estaba afectado de forma leve (C1, C2, C5, C6, C7, T12, L4, L5). Se registró un nódulo de Schmorl en cuerpo central superior de la T12 de intensidad leve (Fig. 3.12). En los miembros superiores se registró osteoartrosis leve en la carilla articular proximal de la $2^{\circ}$ falange derecha del pulgar, asociado a una extensión de superficie articular (ver más adelante). En los miembros inferiores si se registraron proliferaciones periarticulares leves en la cadera izquierda (acetábulo) (Fig. 3.13), leves en rodillas (epífisis distal de fémures y epífisis proximal de tibias de ambas lateralidades) (Fig. 3.8) y leve a moderado en tobillo-pie (calcáneo derecho con proliferaciones leves e izquierdo con grado moderado, al igual que el $1^{\circ}$ metatarso izquierdo y $1^{\circ}$ falange derecho) (Fig. 3.9). Además, el astrágalo y el $1^{\circ}$ metatarso izquierdo presentaban una lesión osteolítica de $1 \mathrm{~cm}$ de radio cada uno con macroporosidad en su base compatible con osteocondritis disecante (Fig. 3.10 y 3.11).

Se hallaron otros indicadores de estrés funcional como fracturas premortem, extensión de carilla articular y patologías vertebrales (Fig. 4). Se registró la presencia de una extensión de faceta articular proximal en $2^{\circ}$ falange correspondiente al pulgar derecho (junto a enfermedad degenerativa articular) (Fig. 4.1). Dos callos óseos en la porción tercio-distal de los $2^{\circ}$ y $3^{\circ}$ metatarsos derechos fueron interpretados como fracturas premortem (Fig. 4.2). En la porción lumbar se observó un aplastamiento de la región anterior del cuerpo vertebral de la L2 como resultado de fracturas por compresión de fuerza axial (Fig. 4.3). Finalmente, el arco neural y el cuerpo de la L5 estaban separadas pero con evidencia de remodelado, por lo se trataba de una fractura premortem, condición conocida como espondilólisis o defecto óseo del arco neural posterior (Fig. 4.4).

Los índices diafisiarios externos, reflejan mayor asimetría en el índice mérico izquierdo (13,6\%), seguido del índice cnémico derecho (3,9\%), índice pilástrico derecho $(1,7 \%)$ e índice diafisiario del húmero izquierdo (1,2\%). El ángulo de torsión femoral refleja un notorio grado de anteversión en ambos lados, pero particularmente en la cadera izquierda $\left(18^{\circ}\right)$ (Tabla 1$)$.

Finalmente, se hallaron marcas de corte posiblemente perimortem (Fig. 5). En la $7^{\circ}$ costilla derecha presentaba dos marcas de tipo cortante lineales y paraleles de 4 a $5 \mathrm{~mm}$ de largo en la superficie dorsal (Fig. 5.1). Por su parte, en la superficie superior del cuerpo de la L4, se 


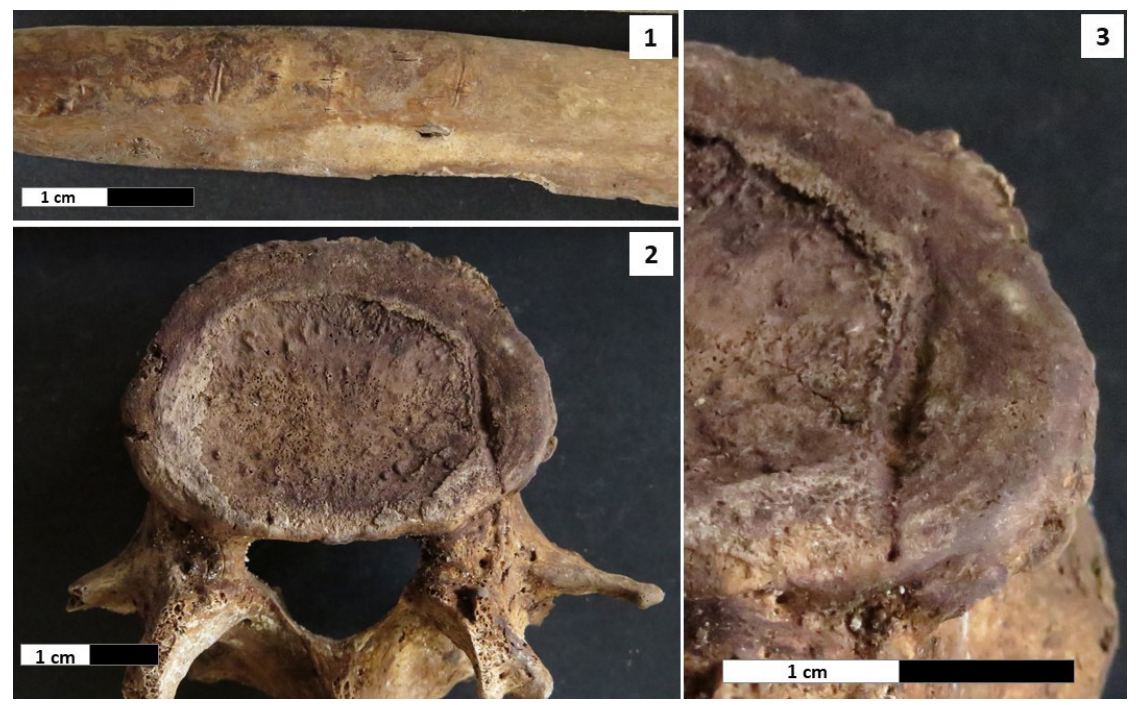

Figura 5. Marcas de cortes perimortem. Referencia: 1, dos marcas de tipo cortantes paralelas de 4 a $5 \mathrm{~mm}$ de longitud en superficie interna de cuerpo de $7^{\circ}$ costilla derecha; 2, vértebra lumbar (L4) con una marca de tipo cortopunzante en forma de $\checkmark$ en dirección anteroposterior en línea recta a la altura del proceso transversal derecho; 3 , detalle de la marca.

Figure 5. Perimortem cut marks. Reference: 1, two parallel cutting type marks 4 to 5 $\mathrm{mm}$ long on the internal surface of the body of the 7th right rib; 2, lumbar vertebra (L4) with a $\mathrm{V}$-shaped sharps-type marking in an anteroposterior direction in a straight line at the level of the right transverse process; 3 , mark detail.

registró una marca de tipo cortopunzante en forma de $\checkmark$ en dirección anteroposterior en línea recta a la altura del proceso transversal derecho (Fig. 5.2 y 5.3 )

\section{Discusión}

\section{Procesos postmortem}

La cultura material asociada, el patrón de entierro, la integridad y la representación esqueletal del individuo sugieren una inhumación originaria en tierra. El patrón secundario y desarticulado de los huesos indica que el entierro original fue perturbado y reubicado en un nuevo lugar (sepultura actual). Este proceso generó la pérdida de algunos elementos óseos pequeños de manos y pies que posiblemente quedaron en el lugar original, así como también a la incorporación accidental de otros elementos del entorno como el carozo de durazno. Si bien se desconoce la ubicación exacta donde fue inhumado originariamente dentro del cementerio, el 13 de noviembre de 1868 se dispuso la erección de un monumento para depositar los restos del Teniente Coronel en la entrada del cementerio de Mendoza (Sosa, 2015), el cual ya se encontraba construido para el año siguiente en su actual ubicación, según afirma Manuel Estrada (Saldaña, 1939). De esto se puede interpretar que el cuerpo se mantuvo inhumado poco menos de nueve años y tras su exhumación (entre fines de 1868 y 1869) (Saldaña, 1939; Sosa, 2015), los restos fueron reducidos a una urna o ataúd pequeño (al cual corresponde la madera y herrajes hallados). Otros indicadores como el resquebrajamiento del tejido cortical de huesos largos y tabla externa del cráneo y un depósito de cristales minerales (blanco translúcido) pueden estar respondiendo a las condiciones del ambiente de los restos una vez que fueron depositados y sellados dentro del monumento mortuorio, caracterizado por oscuridad, humedad y nula circulación del aire.

\section{Perfil osteobiográfico general}

El sexo y el rango de edad de muerte (masculino de 41 a 51 años, con edad promedio en 46,6 años) condicen con el documentado para Ambrosio Sandes, un individuo masculino cuya edad al momento de la muerte varía entre 48 años según Saldaña (1939) y 43 años según la sepultura. Si bien no se dispone de datos precisos sobre su estatura y masa corporal, los resultados obtenidos mediante el estudio osteológico $(177,4 \mathrm{~cm}$ promedio de alto y de $70 \mathrm{~kg}$ promedio de peso) guarda relación con las referencias históricas que mencionan que era una persona corpulenta (Mercado Luna, 1974) y de elevada estatura (Cuestas, 1897). Incluso, si se compara con las estaturas calculadas en muestras de individuos masculinos de las poblaciones coloniales de Mendoza (siglo XVIII y XIX), los restos analizados presentan valores por encima del límite máximo registrado de 166,19 cm (Mansegosa, 2015:171).

En relación con la ascendencia biológica, las características morfológicas del cráneo lo posicionan dentro del grupo europeo. Sin embargo, el índice subtrocantérico lo clasifica como amerindio. Los rasgos morfológicos intermedios pueden estar indicando una ascendencia mixta (Burns, 2012), lo cual coincide con los documentos históricos que sostienen que el padre era de origen europeo mientras que la madre era nativa o mestiza (Cuestas, 1897).

\section{Perfil osteobiográfico específico: primeros años de vida y adultez}

Los primeros años de vida del individuo, particularmente antes de los 4 años de edad, estuvieron marcados por episodios de estrés-metabólico nutricional que llegaron a afectar todo el organismo. Así lo sugieren las líneas de hipoplasia dental, que son resultado de una disrupción en el proceso de formación del esmalte (amelogénesis). Su presencia se asocia a múltiples causas (i.e. deficiencias en la dieta de micronutrientes, traumas del nacimiento, enfermedades gastrointestinales) (Goodman y Rose, 1990; Ritzman, Baker y Schwartz 2008), aunque se ha demostrado que los defectos del esmalte son más frecuentes en poblaciones con mayor exposición a enfermedades y disminución del acceso a los alimentos 
(Larsen y Hutchinson, 1992). Si bien es un indicador no específico de estrés ambiental, da cuenta condiciones generales en las que el individuo creció y se desarrolló.

La dieta desde la pubertad y toda su adultez, en función de los indicadores de salud oral, sugiere una reducida ingesta de alimentos cariogénicos (propio de dietas agrícolas o mixtas) (i.e. Larsen, 2015). Si bien la pérdida postmortem de piezas dentales puede estar sesgando esta interpretación, se ha registrado que el consumo de carne y dietas más fibrosas producen un desgaste más intenso y de tipo plano (Hillson, 2002; Smith, 1984), como las que se registró. El individuo analizado desarrolló gran parte de su vida en el ámbito rural y cuya actividad principal era la ganadería, por lo que es esperable que haya tenido una dieta con mayor proporción de consumo cárnico y no tanto de productos agrícolas y derivados procesados. En cuanto a la salud oral, la presencia de periodontitis y sarro en las piezas dentales da cuenta de una deficitaria higiene (Hillson, 2002), esta situación es esperable para el periodo en el que vivió (Gaitán Ammann, 2001-2002).

Los indicadores de estrés funcional permiten indagar sobre el desarrollo de sus principales actividades cotidianas en la adultez, evaluado a partir de la porción anatómica, frecuencia, intensidad o severidad y la función de cada componente del sistema locomotor afectado en base a literatura sobre anatomía clínica y funcional (Cerda Leal, 2012; Ruiz y Latarjet, 2011). De todos los indicadores relevados, los CE (modificaciones de regiones del tejido óseo donde se adhieren músculos, tendones y ligamento dando cuenta del uso y magnitud de las contracciones musculares repetitivas durante la ejecución de las rutinas) (Jurmain, Alves Cardoso, Henderson y Villotte, 2012; Villotte y Knüsel, 2013) y EDA (cambios morfológicos -destructivos y de formación ósea- en la superficie articular como consecuencia de situaciones crónicas de estrés mecánico) (Luna et al., 2017; Waldron, 2009) fueron los que mayor información aportaron a la reconstrucción de patrones generales del uso del cuerpo, aunque las interpretaciones deben ser tomadas con cautela debido a la influencia de múltiples factores (Jurmain, et al., 2012; Villotte y Knüsel, 2013).

En la mitad superior del cuerpo (miembros superiores, columna y cintura escapular) el individuo ejecutó movimientos repetitivos y asimétricos de brazos en acciones como la flexión, extensión y supinación del antebrazo izquierdo y abducción del brazo derecho con micro traumas repetitivos y macro traumas abruptos (Hawkey y Merbs, 1995; Henderson et al., 2015). Los CE en la porción torácica (T6 y T7) y en la costilla derecha sugieren movimiento de rotación de la escápula y la acción de tirar de ella hacia la columna vertebral como así también la expansión y contracción de la parrilla costal derecha (en el recambio de aire durante la actividad física). Los movimientos del brazo derecho pueden asociarse con la funcionalidad de la mano de igual lateralidad $\left(2^{\circ}\right.$ falange) por la extensión de superficie articular ante la presión de fuerzas opuestas (Estévez González, 2002), la cual sugiere una acción prolongada en el tiempo del dedo pulgar presionando algún objeto en dirección medial. Si bien esta patología se registra comúnmente en el pie (falanges y metatarsos ante acuclillamiento o arrodillado) (i.e. Estévez González, 2002; Serrano y Santos, 2014) es posible que haya sido como resultado de tener la mano derecha cerrada para sostener un objeto, que en este caso puede ser interpretado como la empuñadura de la espada o una lanza (Cuestas, 1897; Sarmiento, 2014 [1865]). La mayor frecuencia de EDA en la porción cervical de la columna no responde a una relación normal de fuerza ya que es esperable una mayor afectación en la columna lumbar (Argoff y Wheeler, 1998). Por ende, pudo haber llevado soporte de cargas en la cabeza o adoptados movimientos de ligeras flexiones repetitivas y/o de larga permanencia en posición sentada ( $\sin$ apoyo en un respaldo), lo que ha sido documentado en cargadores, zapateros, trabajadores manuales y soldados (Casas Flores, 1997). El nódulo de Schmorl hallado a nivel de la región torácica de la columna vertebral consiste en una lesión osteolítica generada en las carillas articulares de los cuerpos vertebrales vinculada a una compresión intensa y gradual, resultado de requerimientos biomecánicos excesivos en la región torácica baja por fuerza de compresión axial y torsión, aunque no deben descartarse otros factores (i.e. morfológicos, edad, defectos congénitos y alteraciones metabólicas) (Jurmain et al., 2012; Pfirrmann y Resnick, 2001; Suby, 2014). Las actividades agrícolas-ganaderas que implicaron carga y/o traslado de peso ejerciendo fuerzas de compresión axial o torsión de dicha porción (Henríquez y Arriaza, 2013; Pfirrmann y Resnick, 2001), como así también el impacto repetitivo generado por la vibración del caballo y microtraumatismos por repetición (Hobbs et al., 2014; Wentz y De Grummond, 2009), fueron actividades iniciadas por Sandes en la infancia y que se intensificaron en la vida adulta por su carrera militar. Éstas pudieron ser la causa de dicha lesión, sobre todo si se considera una mayor influencia de su manifestación ósea cuando las actividades físicas se realizan en edades tempranas (desde los 9 años) (Dar et al., 2010). La fractura del cuerpo de la L2 está asociada a la compresión del cuerpo vertebral por levantamiento de cargas pesadas, una caída desde una altura y un golpe en la espalda o los hombros, con mayor predisposición ante la presencia de osteoporosis (Jiménez Brobeil, Oumaoui y Souich, 2010). La espondilólisis de la L5, está asociada principalmente al estrés funcional como resultado de prácticas de actividades físicas repetitivas intensas, por sobrecarga o trauma agudo (i.e. caída), aunque existe una predisposición genética (Stroe et al., 2014; Waldron, 2009). Es una patología más frecuente entre atletas, gimnastas de alto rendimiento, bailarines y militares, todos ellos en acciones como levantamiento de pesas, la lucha, el remo, el lanzamiento de objetos y 
montar a caballo (Pugh y Bolin, 2004; Stroe et al., 2014; Waldron, 2009).

En la mitad inferior del cuerpo (miembros inferiores y cintura pélvica), se observó una disminución de frecuencia y asimetría de CE pero un aumento de lesiones en articulaciones (EDA y OD) y fracturas en diáfisis. Se destacan movimientos de flexión de la rodilla (acercando la pierna en dirección de la nalga), la rotación medial de la rodilla y extensión de la cadera hacia atrás (en dirección de la espalda), según el desarrollo del $m$. semimebranoso. El CE correspondiente al ligamento calcaneoperoneo es escasamente estudiado, pero tiene una significativa relevancia para la estabilidad del tobillo ya que mantiene su tensión permanente en el movimiento de flexión, extensión y la posición de varo del pie (Golanó Álvarez, Pérez-Carro, Saenz y Vega, 2004:40); es decir, con la planta del pie hacia dentro, apoyando el borde externo y los dedos anular y meñique, con posible lateralización de las piernas y rodillas. Los movimientos sugeridos de ambos CE pueden asociarse a las actividades de locomoción (caminata en terrenos desnivelados) y/o la montura en caballo, ya que las fuerzas aplicadas en los estribos de los caballos y contracción de la pierna en dirección medial para la estabilidad durante la montura son compatibles; sin embargo, ya que el ligamento calcaneoperoneo no es un CE comúnmente relevado, el movimiento interpretado debe ser tomado con recaudo. La denominada "posición del jinete" genera cambios morfológicos óseos específicos, algunos de los cuales han sido relevados en el individuo y que tienen en común la asimetría en la demanda biomecánica de las articulaciones y complejos musculares de cintura pélvica y miembros inferiores (Anđelinović, Anterić, Škorić y Bašić, 2015; Auvinet, 1999; Berthon et al., 2019; Hobbs et al., 2014; Larentis, 2017): mayor ángulo de anteversión del fémur izquierdo (cuello femoral izquierdo más inclinado hacia adelante y rotado medialmente en relación al derecho), aumento en el índice subtrocantérico (13,6\% más que el derecho), cadera izquierda con EDA (osteofito leve) y cambio morfológico con ovalamiento vertical del acetábulo, tobillo-pie izquierdo con EDA (osteofitos moderados) y OD, patología caracterizada por la separación localizada de un fragmento del cartílago articular y el hueso subcondral como resultado de microtraumatismos reiterados (influenciado por otros factores como los genéticos-hereditarios, variaciones anatómicas de las articulaciones, isquemia del cartílago durante el crecimiento o la vejez de un individuo) (Zuñiga Thayer et al., 2018). Esta distribución de indicadores en la cadera y pierna sugiere movimientos y posiciones repetitivas del cuerpo sobre un solo lado, lateralización que es atribuida entre los jinetes como compensación para disipar el dolor o resultado de utilización de armas (i.e. arco, espadas, lanzas) montado en el caballo (Anđelinović et al., 2015; Auvinet, 1999; Berthon et al., 2019; Hobbs et al., 2014; Larentis, 2017).
En cuanto a las fracturas premortem en la $2^{\circ}$ y $3^{\circ}$ metáfisis distal de metatarsos derechos, las mismas responden a un evento traumático por una carga de fuerza por encima de la capacidad de resistencia del hueso que dio como resultado una discontinuidad parcial o completa. Están asociadas a situaciones accidentales (i.e. caída en altura impactando primero con los pies) o de violencia (i.e. un objeto contundente), aunque muchas lesiones traumáticas pueden producirse con bajas cargas de fuerza ante osteoporosis, enfermedades congénitas e infecciosas (Waldron, 2009).

La reacción perióstica es una respuesta inflamatoria del periostio ante diferentes causas y cuya manifestación consiste en placas óseas con márgenes demarcados o elevaciones irregulares en la superficie del hueso (Waldron, 2009; Weston, 2012). Es un indicador de salud inespecífico y puede ser causado por traumas, infecciones crónicas, hemorragias, tumores, condiciones congénitas, hipervitaminosis A, entre otros (Waldron, 2009). La distribución, localización y características morfológicas de las lesiones sugiere la exposición a eventos infecciosos no sistémicos que fueron todos superados, con excepción de uno que aún estaba activo en la mano derecha. Si se considera dicha distribución en relación con algunas marcas aún visibles en la piel del daguerrotipo de 1861 y la cantidad de escenarios bélicos en los que participó (con exposición a golpes con objetos contundentes, impacto de proyectil, cortes de lanza, facón o espada), no es arriesgado proponer que la mayoría de ellas podría corresponder con traumatismos. Incluso, la presencia de reacción perióstica en miembros inferiores (la mitad de elementos afectados con dicha patología) ha sido registrado entre jinetes (Anđelinović et al., 2015).

Tal vez uno de los aspectos menos concordantes a nivel osteobiográfico en relación al personaje de Sandes tiene que ver con la Hemangioma en el frontal del cráneo, patología de la cual no se halló ninguna mención en las biografías y literatura consultadas. Se trata de una neoplasia o tumor benigno que en casos clínicos modernos excepcionalmente afecta a los huesos craneales $(0,2 \%$ del total de neoplasias) y que se originan en los vasos intrínsecos de los tejidos (cavernosos y/o vasculares). Invade el diploe manifestándose en la tabla externa (y/o interna) como erosiones corticales líticas, ocasionalmente acompañadas de reacción perióstica (Haeren et al., 2012; Nasser et al., 2007; Vega et al., 2010). Un hemangioma cavernoso como la que presenta el individuo, tuvo que haberse plasmada ante el ojo de sus coetáneos como un abultamiento en la frente de Sandes (Haeren et al., 2012; Vega et al., 2010). Sin embargo, la ausencia de referencias de este aspecto físico notorio genera dos posibilidades: los restos no pertenecen a Ambrosio Sandes o la patología se manifestó de forma rápida en los últimos años de su vida, acompañado por algún ocultamiento intencional. En relación a esta última opción, casos clínicos en pacientes vivos reflejan la rapidez de su manifestación y apariencia 
física: pacientes entre 37 y 59 años que atestiguan un aumento de volumen en región frontal próxima al borde orbital incolora sobre la piel, indolora y de rápido desarrollo (en menos de un año ya adoptaba dimensiones similares a la del individuo analizado) (Nasser et al., 2007; Vega et al., 2010). Se desconoce la patogenia, pero se cree que puede ser congénito o relacionado con algún traumatismo previo (Vega et al., 2010), siendo este último la principal etiología en revisión de literatura reciente (Haeren et al., 2012).

El último suspiro: hipótesis sobre las causas de muerte. La muerte del coronel Sandes no está aclarada y existen varias hipótesis. Una de las que tiene mayor peso es la que afirma la muerte como consecuencia de una grave herida en un atentado perpetrado hacia él días antes de su muerte y de la que no se recuperó. Quiroga (1975), historiador mendocino que relata el episodio en base a la obra de Dalmiro Adaro (Reminicencias históricas de San Luis 1815-1861), sitúa a Ambrosio Sandes saliendo de una pulpería de la ciudad de San Luis, donde residía, y encontrándose con una persona en aparente estado de ebriedad. El relato continúa de la siguiente forma:

"Al lado de esa pila, fingiendo distracción o embriaguez, ocupado en el centro de la acera se hallaba una persona en traje de gaucho y Sandes cree que la actitud del gaucho es un acto de descomedimiento a su persona, intenta darle un empellón para que le deje libre el paso, pero el desconocido paisano, con rapidez inesperada, esgrime un puñal de regulares dimensiones y le aplica un golpe de punta en el costado derecho que le secciona una o dos costillas, y va a incrustársele en una vértebra en ese costado. El puñal se rompe y el heridor se queda solamente con el cabo en la mano, con una S de cobre que le sirve de guarnición, y Sandes, con lo menos veinte centímetros de la hoja en el interior del cuerpo" (Quiroga, 1975).

Dos días después de este episodio el Coronel Sandes, muy mal herido, se dirigió a la ciudad de Mendoza designado al regimiento $1^{\circ}$ de esa provincia bajo las órdenes del comandante Ignacio Segovia. Es aquí cuando los testimonios mencionan otro episodio que ofrece una segunda hipótesis de la razón de su muerte: envenenamiento durante un banquete en la quinta de la familia Recuero (Luján de Cuyo, Mendoza) donde participaron algunos funcionarios del gobierno mendocino y Sandes (Campano, 2017). Al día posterior, tres de los presentes (el gobernador Luis Molina, Ambrosio Sandes y el capitán Ramírez) tuvieron síntomas similares: dolor de estómago, vómitos y un profundo deterioro corporal. Ambrosio Sandes, que se encontraba en grave estado en la quinta de Villanueva, falleció el 15 de setiembre a las ocho de la noche. Unos días más tarde falleció Luis Molina y el capitán Ramírez (Campano, 2017). Si bien el autor de esta nota no ofrece documentación respaldatoria de estos hechos, tampoco se ha realizado un análisis químico específico en los tejidos para evaluar la presencia de sustancias.

Si consideramos que la marca de corte perimortem en superficie superior del cuerpo de la L4 corresponde al ingreso de un objeto cortopunzante con filo en la región dorsal derecha del mismo, podemos sugerir una relación entre el objeto (arma blanca), lateralidad (derecha) y porción anatómica (torso y columna) mencionada por Quiroga (1975). Sin embargo, se esperaría una lesión similar en la superficie contigua a la vértebra (cara inferior de L3) que no se halló. Por otro lado, las marcas en superficie dorsal de la $7^{\circ}$ costilla derecha resultan difíciles de atribuir a este episodio ya que es poco probable que el arma haya ingresado sin afectar otra costilla. Sin dejar de lado estos interrogantes, la causa de muerte del individuo puede ser asociada con la lesión detectada en la vértebra como así también otras que pudieron afectar tejidos blandos (entre ellos, algunos órganos vitales) pero que resultan imperceptibles en los huesos.

\section{Conclusiones}

Los restos humanos analizados en la sepultura de Ambrosio Sandes permitieron obtener una caracterización del perfil osteobiográfico general cuyos resultados son concordantes con el perfil biográfico brindado por la historiografía, mientras que el perfil individualizante brindó información significativa que no pudo ser contrastada debidamente ante la ausencia y/o ambigüedad de la información documental-histórica referida al personaje estudiado. Más allá de esto, el enfoque osteobiográficohistórico permitió interpretar los resultados de los bioindicadores de salud, dieta y estrés funcional de acuerdo a las distintas etapas de su vida (infancia, carrera militar y los eventos próximos a su fallecimiento) en función del contexto cultural del período en que vivió. Uno de los rasgos característicos del esqueleto, que coincide con el de Ambrosio Sandes, es la marcada exposición a episodios de violencia interpersonal y de elevado compromiso del cuerpo en la actividad física del individuo (fuerte correlato con la actividad militar). Por ende, la asociación de estos bioindicadores con estilos de vida (en general) y eventos (en particular) de relevancia en su trayectoria de vida a partir de hipótesis de base documental sobre su estado de salud y causa de muerte, fueron altamente concordantes.

En este trabajo, la bioarqueología (a partir del enfoque osteobiográfico) permitió ser una vía complementaria para discutir hechos del pasado aun estando documentados. Por un lado, muestra la factibilidad de hallar rasgos o particularidades fisiológicas de personajes que no están registradas por sus contemporáneos o quienes relatan el pasado (i.e. neoplasia). Por el otro, ofrece un modo diferente de conocer el proceso de consolidación del Estado Argentino desde la perspectiva de la salud, es decir, cómo impactaron las conductas realizadas en vida 
y que se han plasmado en el cuerpo de los protagonistas.

Considerado como estudio de caso, el análisis realizado es una demostración del potencial de las estrategias metodológicas que puede emplear la antropología forense, particularmente en líneas de análisis escasamente explotadas (i.e. indicadores de estrés funcional para la reconstrucción de patrones generales del uso del cuerpo como un elemento probatorio más de identificación).

Finalmente, destacamos que los resultados forman parte de una larga y fructífera interacción entre la academia y el municipio al abordar tres ejes que se retroalimentan: la conservación patrimonial, la investigación y la difusión del conocimiento científico generado a partir de la incorporación en el guion de recorridos turísticos dentro del cementerio promocionado por el Municipio de Capital.

Mendoza, 4 de mayo de 2020

\section{Agradecimientos}

Al personal del Cementerio Municipal de Capital y a su director, José Curia, por la predisposición permanente a colaborar y trabajar en conjunto. A la municipalidad de la Ciudad de Mendoza, por permitir el ingreso y las autorizaciones correspondientes. $Y$ a los evaluadores del presente trabajo por enriquecer, desde diferentes campos disciplinares, la información presentada.

\section{Bibliografía}

Anđelinović, Š., Anterić, I., Škorić, E. y Bašić, Ž. (2015). Skeleton Changes Induced by Horse Riding on Medieval Skeletal Remains from Croatia. The International Journal of the History of Sport. DOI: 10.1080/09523367.2015.1038251

Argoff, C. E. y Wheeler, A. H. (1998). Trastornos del dolor espinal y radicular. Clínicas neurológicas, 16 (4):833-849.

Auerbach, B. M y Ruff, C. B. (2004). Human Body Mass Estimation: A Comparison of Morphometric and Mechanical Methods. American Journal of Phisycal Anthropology, 125:331-342.

Auvinet, B. (1999). Lombalgies et équitation. Synoviale, 83:25-31.

Bass, W. M. (1995). Human osteology: A laboratory and field manual of the human skeleton. Missouri Arch. Society, Special Public. $N^{\circ} 2$, Columbia.

Buikstra, J. E. y Ubelaker, D. H. (1994). Standards for Data Collection from Human Skeletal Remains. Arkansas Archaeological Survey Research Series N44, Arkansas.

Berthon, W., Tihanyi, B., Kis, L., Révész, I., Coqueugniot, H., Dutour, O. y Pálfi, G. (2019). Horse riding and the shape of the acetabulum: Insights from the bioarchaeological analysis of early Hungarian mounted archers (10th century). International Journal of Osteoarchaeology, 29 (1):117-126. DOI: 10.1002/oa.2723

Burns, K. M. (2012) Forensic Anthropology Training Manual. Routledge. New York.

Campano, C. (2017). Magnicidio a la Mendocina. Diario LoS Andes. https://www.losandes.com.ar/article/magnicidio-ala-mendocina

Byers, S. (2002). Introduction to forensic anthropology: A textbook. Allyn \& Bacon, Boston.

Casas Flores, M. J. (1997). Principales marcadores óseos macroscópicos de estrés físico en poblaciones humanas: Su validez como indicadores de gestos repetitivos [Tesis de doctorado no publicada]. Departamento de Biología Animal I (U.D. de Antropología), Universidad Complutense.

Cerda Leal, F. (2012). Guía de Apoyo Anatomofisiología Músculos. Facultad de Educación Pedagogía en Educación Diferencial. Universidad Pedro de Valdivia, Valdivia.

Chávez, F. (1975). General Angel Vicente Peñaloza, el Chacho. Cuadernos de Crisis. Editorial del Noroeste, Buenos Aires.

Codesido, L. (2016). Armar al Estado, construir la Nación. La nacionalización de las fuerzas armadas en la Argentina y su vinculación con el proceso de construcción del Estado argentino entre 1862 y 1880 [Tesis de doctorado no publicada]. Facultad de Humanidades y Ciencias de la Educación, Universidad Nacional de La Plata.

Cuarterolo, M. A. y Alexander, A. (2001). Soldados, 18481927. Edivern, Buenos Aires.

Cuestas, J. L. (1897). Páginas sueltas. Tomo I. Dornaleche y Reyes, Montevideo.

Dar, G., Masharawi, Y., Peleg, S., Steinberg, N., May, H., Medlej, B., Peled, N. y Hershkovitz, I. (2010). Schmörl's nodes distribution in the human spine and its possible etiology. European Spine Journal, 19 (4):670-675. DOI:10.1007/s00586-009-1238-8

Del Río Muñoz, P. (2000). Estudio antropológico-forense, antropométrico y morfológico, de la colección de la Escuela de Medicina Legal de Madrid [Tesis de doctorado no publicada]. Departamento de Medicina Legal, Universidad Complutense.

Elder, G., Johnson, M. K., y Crosnoe, R. (2003). The Emergence and Development of Life Course Theory. Lee, J., J. T. Mortimer y M. J. Shanahan (eds.), Handbook of the Life Course, pp. 3-19. Plenum Press, New York.

Estévez González, M.C. (2002). Marcadores de estrés y actividad en la población guanche de Tenerife [Tesis 
de doctorado no publicada]. Departamento de Historia, Antropología e Historia Antigua, Universidad de La Laguna.

Fernandez-Jalvo, Y. y Andrews, P. (2016). Atlas of taphonomic identifications: 1001+ images of fossil and recent mammal bone modification. Springer.

Gaitán Ammann, F. (2001-2002). Recordando a los Uribe. Memorias de higiene y de templanza en la Bogotá del Olimpo Radical (1870-1880). Revista de Antropología y Arqueología. Volumen Especial. Objetos y relatos: estudios de cultura material, 13:125-146.

Garavaglia, J. C., Ruiz, J. P. y Zimmermann, E. (2012). Las fuerzas de guerra en la construcción del Estado. América Latina, siglo XIX. Prohistoria Ediciones, Rosario.

Gilbert, R. y Gill, G.W. (1990). A metric technique for identifying American Indian femora. Gill, G.W. y S. Rhine (eds.), Skeletal attribution of race, pp. 97-99. Maxwell Museum of Anthropology, Albuquerque.

Glencross, B. A. (2011). Skeletal injury across the life course. Agarwal, S. C., y B. S. Glencross (eds.), Social bioarchaeology, pp 390-409. Wiley-Blackwell, West Sussex.

Golanó Álvarez, P., Pérez-Carro, L., Saenz, I. y Vega, J. (2004). Anatomía de los ligamentos del tobillo. Revista de Ortopedia y Traumatología, 48 (3):35-44.

Goodman, A. H. y Rose, J. (1990). Assessment of systemic physiological perturbations from dental enamel hypoplasias and associated histological structures. Yearbook of Physical Anthropology, 33: 59-110. DOI: 10.1002/ajpa.1330330506

Haeren, R. H., Dings, J., Hoeberigs, M. C., Riedl, R. G. y Rijkers, K. (2012). Posttraumatic skull hemangioma: case report. Journal of neurosurgery, 117 (6):1082-1088.

Hawkey, D. y Merbs, C. (1995). Activity-induced musculoskeletal stress markers (MSM) and subsistence strategy among ancient Hudson Bay Eskimos. International Journal of Osteoarchaeology, 5:324-338.

Henderson, C., Mariotti, V., Pany-Kucera, D., Villotte, S. y Wilczak, C. (2015). The New 'Coimbra Method': A Biologically Appropriate Method for Recording Specific Features of Fibrocartilaginous Entheseal Changes. International Journal of Osteoarchaeology. DOI: 10.1002/oa.2477

Henríquez, M. y Arriaza, B. (2013). Distribución y frecuencia de Nódulos de Schmörl en la columna vertebral de poblaciones prehispánicas de Arica: ¿Indicadores de la carga laboral? Chungará (Arica), 45 (2):311-319.

Hillson, S. (2002). Dental Anthropology. 3rd edition, Cambridge University Press, Cambridge.

Hobbs, S. J., Baxter, J., Broom, L., Rossell, L. A., Sinclair, J. y Clayton, H. (2014). Posture, flexibility and grip strength in horse riders. Journal of human kinetics, 42 (1):113-125.

Jiménez-Brobeil, S. A., Oumaoui, I. A. y Souich, P. D. (2010). Some types of vertebral pathologies in the Argar Culture (Bronze Age, SE Spain). International Journal of Osteoarchaeology, 20 (1):36-46.

Jurmain, R., Alves Cardoso, F., Henderson, C. y Villotte, S. (2012). Bioarchaeology's Holy Grail: The Reconstruction of Activity. Grauer A. L. (ed.), A Companion to Paleopathology, pp: 531-552. Blackwell, West Sussex.

Krenzer, U. (2006). Compendio de Métodos Antropológico Forenses para la Reconstrucción del Perfil Osteobiológico. Centro de Análisis Forense y Ciencias Aplicadas (CAFCA), Serie de Antropología Forense, Guatemala.

Lamendin H., Baccino, E., Humbert, J. F., Tavernier, J. C., Nossintchouk, R. M. y Zerilli, A. (1992). A simple technique for age estimation in adult corpses: the two criteria dental method. Journal of Forensic Sciences, 37 (5): 1373-1379

Larentis, O. (2017). San Martino di Lundo (Trento) Grave 1. Case study of an individual introducing possibilities markers of horse riding.Medicina, 1 (2):00-00.

Larsen, C. S. y Hutchinson, D. L. (1992). Dental evidence for physiological disruption: biocultural interpretations from the Eastern Spanish Borderlands, USA. Journal of Paleopathology, Monographic Publications, 2:151-169.

Larsen, C. S. (2015). Bioarchaeology: interpreting behavior from the human skeleton. Cambridge University Press, Cambridge. DOI: 10.1017/CBO9781139020398

Lovell, C. (2008). Analysis and Interpretation of Skeletal Trauma. Katzenberg, M. y S. Saunders (eds.), Biological Anthropology of the Human Skeleton, pp. 341-386. Wiley Liss, Nueva Jersey.

Luna, L., Aranda, C. y Amorim Alves, A. (2017). Reflexiones sobre el relevamiento y análisis comparativo de patologías osteoarticulares en restos esqueletales humanos. Revista Argentina de Antropología Biológica, 19 (1):7-14.

Mansegosa, D. (2015). Variación morfológica postcraneal en poblaciones históricas del norte Mendoza: análisis de la relación entre la estatura y el índice de platimería. Libro de resúmenes de las XXIV Jornadas de Investigación y VI Jornadas de Posgrado Universidad Nacional de Cuyo. Universidad Nacional de Cuyo, Mendoza.

Mansegosa, D., Giannotti, P. S., Chiavazza, H. y Barrientos, G. (2018). Funciones discriminantes para estimar sexo a partir de huesos largos en poblaciones coloniales del centro oeste de Argentina. Chungara, 50 (1):155-164.

Mansegosa, D., Zorrilla, V., Prieto Olavarría, C. y Giannotti, P. S. (2012). Excavación arqueológica y análisis bioarqueológico en el cementerio de la Capital (Mendoza). Repatriación de 
los restos de Santos Ortiz e Inés Vélez de Ortiz. Revista de Arqueología Histórica Argentina y Latinoamericana, 6:127-156.

Mercado Luna, R. (1974). Los coroneles de Mitre. Editorial Plus Ultra, Buenos Aires.

Nasser, K., Hayashi, N., Kurosaki, K., Hasegawa, S., Kurimoto, M., Mohammed, A. y Endo, S. (2007). Intraosseous cavernous hemangioma of the frontal bone. Neurologia medico-chirurgica, 47 (11):506-508.

Neves, W. A. (1984). Estilo de vida e osteobiografía: a reconstitucao do comportamento pelos ossos humanos. Revista de Pré-historia, 4:287-291.

Pugh, T. J. y Bolin, D. (2004). Overuse injuries in equestrian athletes. Current Sports Medicine Reports, 3 (6):297-303. DOI:10.1007/s11932-996-0003-6

Quiroga, A. (1975). La muerte del coronel Ambrosio Sandes. Revista de la Junta de Estudios Históricos de Mendoza. Segunda época, 8 (2):777-782.

Ritzman, T. B., Baker, B. J., y Schwartz, G. T. (2008). A Fine Line: A Comparison of Methods for Estimating Ages of Linear Enamel Hypoplasia Formation. American Journal of Physical Anthropology 135:348-361

Ruiz Liard, A. y Latarjet, M. (2011). Anatomía humana. $4^{\circ}$ edición. Médica Panamericana, Buenos Aires.

Saldaña, J. M. F. (1939). El Coronel Ambrosio Sandes. Alrededor de una silueta biográfica. Diario El Día (19331953). https://periodicotribuna.com.ar/1105-22-denoviembre-de-1861.html

Sarmiento, D. F. (2014) [1865]. El Chacho, último caudillo: de la montonera a los Ilanos. $1^{\circ}$ edición. EUDEM, Mar del Plata.

Serrano, L. y Santos, A. L. (2014). Minería en aljustrel (Portugal): salud, enfermedad y actividades en los habitantes del pasado. Jangwa Pana, 13 (1):65-81.

Sjovold, T. (1990). Estimation of stature from long bones utilizing the line of organic correlation. Human Evolution, 5 (5): 431-447.

Smith, B. (1984). Patterns of molar wear in huntergatherers and agriculturalists. American Journal of Physical Anthropology, 63:39-56.

Sosa, E. (2015). Vida y Muerte en Mendoza 1787-1923. El sincretismo Cultural a través de la funebria mendocina. Mendoza. Editorial de la Facultad de Filosofía y Letras de la Universidad Nacional de Cuyo, Mendoza.

Stroe, A., Martínez Blanco, S., Rubio Sanz, M. J., Riñones Mena, E., Alonso Garcia, E. y Velázquez Fragua, P. (2014). Revisión de los hallazgos radiológicos asociados a la espondilólisis. Presentación electrónica en el Congreso de la Sociedad Española de Radiología Médica del 2014. DOI: 10.1594/seram2014/S-0107

Stuart-Macadam, P. (1985). Porotic hyperostosis: representative of childhood condition. American Journal of Physical Anthropology, 66:391-389. DOI: 10.1002/ ajpa.1330660407

Suby, J. (2014). Nódulos de Schmorl en restos humanos arqueológicos de Patagonia Austral. Magallania, 42 (1):135-147

Pfirrmann, C. W. y Resnick, D. (2001). Schmorl nodes of the thoracic and lumbar spine: radiographic-pathologic study of prevalence, characterization, and correlation with degenerative changes of 1, 650 spinal levels in 100 cadavers. Radiology, 219:368-374.

Vega, A., Obieta, E. D., Aguado, G., Esqueda, M., Ruíz, S., Ramirez, E. y Pérez, J. (2010). Hemangioma cavernoso multifocal en cráneo: Revisión de un caso. Neurocirugía, 21 (6):484-490.

Villotte, S. y Knüsel, C. J. (2013). Understanding entheseal changes: definition and lifecourse changes. International Journal of Osteoarchaeology, 23:135-146. DOI: 10.1002/oa.2289

Waldron, T. (2009). Paleopathology. Cambridge University Press, Cambridge.

Wentz, R. K. y De Grummond, N. T. (2009). Life on horseback: palaeopathology of two Scythian skeletons from Alexandropol, Ukraine. International Journal of Osteoarchaeology, 19 (1):107-115.

Wescott, D., Cunningham, D. y Hunt, D. (2014). Temporal trends in femoral diaphyseal torsional asymmetry among the Arikara associated with postural behavior. American Journal of Physical Anthropology, 154 (4):512-524.

Weston, D. A. (2012). Nonspecific infection in paleopathology: interpreting periosteal reactions. Grauer, A. L. (ed.), A companion to paleopathology, pp. 492512. Wiley Blackwell, Chichester.

Zúñiga Thayer, R., Suby, J., Flensborg, G. y Luna, L. (2018). Osteocondritis disecante. Primeros resultados en restos humanos de cazadores-recolectores del Holoceno en Patagonia austral. Revista del Museo de Antropología, 11 (1):107-120. 